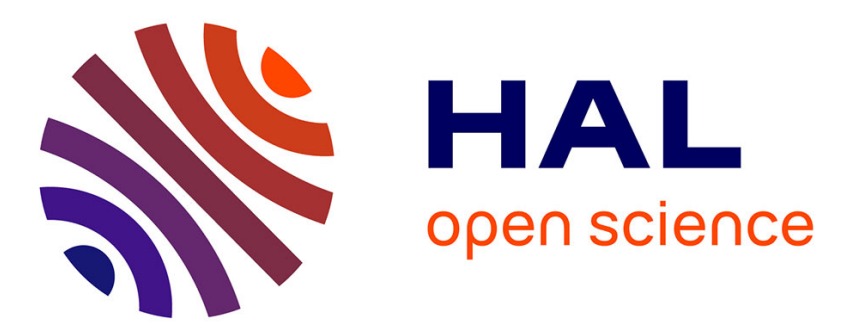

\title{
Fine structure of bright and dark excitons in asymmetric droplet epitaxy GaAs/AlGaAs quantum dots
}

\author{
H. Mekni, A. Pankratov, S. Ben Radhia, K. Boujdaria, M. Chamarro, C. \\ Testelin
}

\section{- To cite this version:}

H. Mekni, A. Pankratov, S. Ben Radhia, K. Boujdaria, M. Chamarro, et al.. Fine structure of bright and dark excitons in asymmetric droplet epitaxy GaAs/AlGaAs quantum dots. Physical Review B, 2021, 103 (7), 10.1103/PhysRevB.103.075302 . hal-03156614

\section{HAL Id: hal-03156614 https://hal.science/hal-03156614}

Submitted on 2 Mar 2021

HAL is a multi-disciplinary open access archive for the deposit and dissemination of scientific research documents, whether they are published or not. The documents may come from teaching and research institutions in France or abroad, or from public or private research centers.
L'archive ouverte pluridisciplinaire HAL, est destinée au dépôt et à la diffusion de documents scientifiques de niveau recherche, publiés ou non, émanant des établissements d'enseignement et de recherche français ou étrangers, des laboratoires publics ou privés. 


\title{
Fine structure of bright and dark excitons in asymmetric droplet epitaxy GaAs/AlGaAs quantum dots
}

\author{
H. Mekni $\odot,{ }^{1}$ A. Pankratov, ${ }^{2}$ S. Ben Radhia, ${ }^{1}$ K. Boujdaria, ${ }^{1, *}$ M. Chamarro $\odot,{ }^{2}$ and C. Testelin ${ }^{2}$ \\ ${ }^{1}$ Université de Carthage, Faculté des Sciences de Bizerte, LR01ES15 Laboratoire de Physique des Matériaux: \\ Structure et Propriétés, 7021 Zarzouna, Bizerte, Tunisia \\ ${ }^{2}$ Sorbonne Université, CNRS, Institut des NanoSciences de Paris, F-75005, Paris, France
}

(Received 6 August 2020; revised 7 January 2021; accepted 25 January 2021; published 9 February 2021)

\begin{abstract}
We have calculated the exciton fine structure splittings (FSS) of asymmetric GaAs/AlGaAs quantum dots (QDs) obtained after Al droplet epitaxy and subsequent nanoholes formation followed by annealing and GaAs filling of nanoholes. We used a $\mathbf{k} \cdot \mathbf{p}$ model and considered the heavy-hole and light-hole mixing to calculate the electron-hole exchange interaction (EI). The two components, long-range (LR) and short-range (SR) of the EI, were deduced. The exciton fine structure is organized, as usual in zinc-blende compounds, into two groups of states: bright (optically active) and dark states. The bright-dark and bright-bright splittings contain LR and SR contributions, the LR part representing 5 to $68 \%$ of the total bright-dark splitting and 69 to $76 \%$ of the total bright-bright splitting for sizes experimentally explored. In QDs having $\mathrm{C}_{2 v}$ symmetry, LR and SR contributions to dark-dark splitting have to be calculated at the second order of perturbation theory. A good agreement between the theory and experiment is obtained for QDs with different degrees of asymmetry, from QD having an isotropic shape to QD with a very anisotropic shape.
\end{abstract}

DOI: 10.1103/PhysRevB.103.075302

\section{INTRODUCTION}

Semiconductor quantum dots (QDs) have been the object of extensive studies, their discrete states making them attractive to quantum-technology applications. Important successes have been obtained like efficient single-photon sources [1,2] or the realization of qubits $[3,4]$. In this framework, confined neutral excitons play a key role, being the first optically excited state. In conventional semiconductors, like GaAs, neutral excitons are splitted in two kinds of states, optically active [bright exciton (BE)] or inactive [dark exciton (DE)]. The BEs have been extensively studied in the last decades because they are decisive for optoelectronic applications based on QDs [5-8].

The DE states appear as promising candidates for the realization of qubits, since they have long life and coherence times, compared to BE states. The writing and control of the DE states have been recently evidenced $[9,10]$. Nonetheless, due to the complexity in the optical initialization and reading processes [11], a precise understanding and modelization of the excitonic states is necessary, particularly the fine structure splittings (FSS) involving DE and BE states.

The most widely studied QDs are grown by molecular beam epitaxy (MBE) and a self-assembly Stranski-Krastanov approach. These QDs present residual strains [12] or unintentional intermixing [13] with the barrier material, affecting their optoelectronic properties. Alternative growths, based on droplet epitaxy, have been developed to realize strain-free QDs. The first approach was to deposit pure material droplets,

*kais.boujdaria@fsb.rnu.tn followed by recrystallization under a complementary compounds flux [14]. More recently, droplet etching has been developed to grow self-assembled nanoholes, subsequently filled to form QDs [15]. This technique leads to highly uniform [16] and isotropic GaAs QDs [17] showing very small bright-bright exciton splitting [18]. This nanohole-infilling approach can also be associated with nanopatterned substrate to control the QDs position and realize QDs arrays $[19,20]$. Moreover, in these filled nanohole QDs, very recent studies have evidenced the opportunity to observe DE states $[21,22]$ to control the DE decay rate and heavy-hole (HH)-light-hole (LH) mixing via strain or electric field [23]. Those QDs can then offer the necessary bricks to a platform with well controlled and uniform qubits, encoded on dark DE states.

In this paper, we propose modeling the electronic and excitonic properties of strain-free GaAs/AlGaAs QDs obtained via nanohole etching. Among the theories currently used to model solid state properties, the $\mathbf{k} \cdot \mathbf{p}$ approach is a well established method to calculate electronic and excitonic states in quantum nanostructures [24]. Other numerical methods are also very developed in quantum nanostructures modelization such as empirical pseudopotentials (PP) and tight-binding (TB) approaches. While sometimes more complete, these approaches may be numerically very costly. In the end 1990s, Fu et al. [25] presented a PP method within local-density approximation to calculate the e-h exchange splitting in bulk semiconductors, with some discrepancies with experiments. In order to improve theoretical and experimental agreement, Luo et al. [26] proposed later a PP approach combined with the configuration interaction (CI) method to calculate the FSS of GaAs QDs. More recently, this PP method, combined with CI method [27] or screened CI method [28], provided accurate 
many-body theoretical calculations with an agreement with experimental measurements, either for InAs [27] or CdSe QDs [28]. The TB method has also been the object of numerous studies. Combining TB approach [29] for single particle and CI method for excitonic properties has permitted to calculate the many-body spectrum of InAs QD [30] and to estimate the QD excitonic fine structure [31]. Zielinski [32] recently improved the CI approximation adopted in the Refs. [30,31] by going beyond $s, p, d$ shells and including the $f$ shells as well. This work provides valuable information into the bright and dark excitonic fine structure for elongated InAs/InP QDs, and its predictions compare well with experimental data.

The $\mathbf{k} \cdot \mathbf{p}$ method has also been largely explored for quantum nanostructures. One can cite the work of Kadantsev and Hawrylak [33] who applied the $\mathbf{k} \cdot \mathbf{p}$ approach to investigate the excitonic fine structure as a function of QD anisotropy, size, and applied electric field. More recently, by combining the $\mathbf{k} \cdot \mathbf{p}$ method for the computation of single-particle states and the CI method for excitonic states, it was possible to investigate the magneto-optical properties excitonic fine structure of GaAs/AlGaAs QDs obtained by droplet-etching method [34,35]. In the following, we will use a $\mathbf{k} \cdot \mathbf{p}$ approach, already used for similar GaAs/AlGaAs QDs [36,37]. We focus our study on the influence of the QD size and shape on the bright-dark (BD), dark-dark (DD), and brightbright (BB) exciton splittings. It is well established that the exciton FSS is controlled by the electron-hole (e-h) exchange coupling, mediated by Coulomb interaction [38,39]. The two main contributions are the short-range (SR) and long-range (LR) coupling, as defined in Refs. [38,39]. These exchange couplings are particularly sensitive to QD anisotropy [40] or strain [41]. Following recent modelizations [36,37] (those works focusing on theoretical study of the electronic states, such as the single particles levels or single particle wave functions), we have derived the $\mathrm{BD}, \mathrm{DD}$, and $\mathrm{BB}$ splittings and compare our theoretical results with very recent microphotoluminescence measurements $[21,22,37]$. The calculated BB splitting is smaller than the experimental one while a good agreement between theory and existing experimental observation is obtained for BD and DD splittings. We also discuss the different contributions to FSS that are not taken into account in this work and could improve agreement between theory and experiment, especially for the BB splitting contributions $[32,42-44]$.

The outline of the paper is the following: In Sec. II, we describe the theoretical methodology followed to obtain different splittings involved in the exciton fine structure. In Sec. III, we apply the developed theoretical method to describe recent microphotoluminescence experiments on the exciton FSS obtained for nanohole-infilled GaAs/ $\mathrm{Al}_{0.45} \mathrm{Ga}_{0.55}$ As QDs. Size and shape QDs parameters were deduced from previous spectroscopic and AFM characterizations. We conclude in Sec. IV.

\section{THEORETICAL METHODOLOGY}

The upper valence band is associated to $\mathrm{HH}$ states $\left|J^{h}=3 / 2, J_{z}^{h}= \pm 3 / 2\right\rangle$ while the lower conductions states are $\left|C_{ \pm}\right\rangle=\left|S^{e}=1 / 2, S_{z}^{e}= \pm 1 / 2\right\rangle$ for the majority of the epitaxial QD made of III-V or II-VI zinc blende semiconductors. After promotion of an electron from the top of the valence
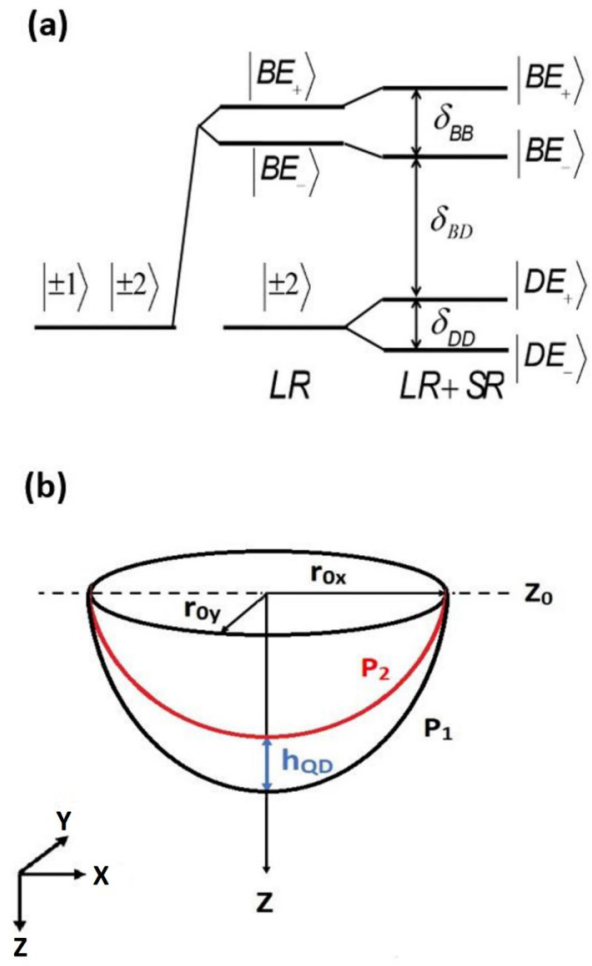

FIG. 1. (a) Energy labeling of the fine structure exciton states. (b) The QD shape and their corresponding parameters.

band to the bottom of the conduction band, four e-h pairs are then possible, commonly divided into bright and dark excitons. The e-h pairs $\left(J_{z}^{h}= \pm 3 / 2, S_{z}^{e}= \pm 1 / 2\right)$ with angular momentum \pm 1 are dipole allowed and are referred to as $\mathrm{BE}$, while the $\left(J_{z}^{h}= \pm 3 / 2, S_{z}^{e}= \pm 1 / 2\right)$ pairs, with angular momentum \pm 2 , are dipole forbidden and referred to as DE.

In the following, we present the theoretical approach used to describe the microelectronvolt scale structure created by spin-dependent interactions between the electron and the hole, that is, the excitonic fine structure. $\left|B E_{+}\right\rangle$and $\left|B E_{-}\right\rangle$are the high and low energy BE states, while $\left|D E_{+}\right\rangle$and $\left|D E_{-}\right\rangle$are the high and low energy DE states. A scheme describing the exciton energy splittings is given in Fig. 1(a).

Following the procedure described in Appendix A and used previously [37], we begin to calculate the single particle energy levels $E_{v}(v=e, h)$ and the single particle wave functions $\Psi_{n}^{v}\left(\mathbf{r}_{v}\right)\left(\mathbf{r}_{v}\right.$ being the coordinates of the $v$ carrier), by solving the single particle Schrödinger equation $\mathcal{H}_{v} \Psi_{n}^{v}\left(\mathbf{r}_{v}\right)=$ $E_{\nu} \Psi_{n}^{v}\left(\mathbf{r}_{v}\right)$ with $\mathcal{H}_{\nu}=\mathcal{T}_{v}\left(\mathbf{r}_{v}\right)+\mathcal{V}_{\nu}\left(\mathbf{r}_{v}\right)$. $\mathcal{H}_{\nu}$ is a single particle Hamiltonian associated to the electron (e) or hole (h), $\mathcal{T}_{v}$ is the kinetic energy operator, and $\mathcal{V}_{v}$ is the confinement potential induced by the band offset. Let us notice that in Ref. [37], we have presented a theoretical model that takes into account the QD shape anisotropy which induces the cylindrical symmetry breaking. The numerical parameters used in our calculations are laid out in Appendix B. The lattice mismatch being very small for $\mathrm{GaAs} / \mathrm{Al}_{0.45} \mathrm{Ga}_{0.55} \mathrm{As} \mathrm{QD}$ ( $0.2 \%$ of mismatch), compare to InAs/GaAs QD (7\% of mismatch), we have neglected any hydrostatic and shear strain and then any piezoelectric effect. QDs shape elongations have a pronounced effect on the exciton fine structure in semiconductor QDs and have been 


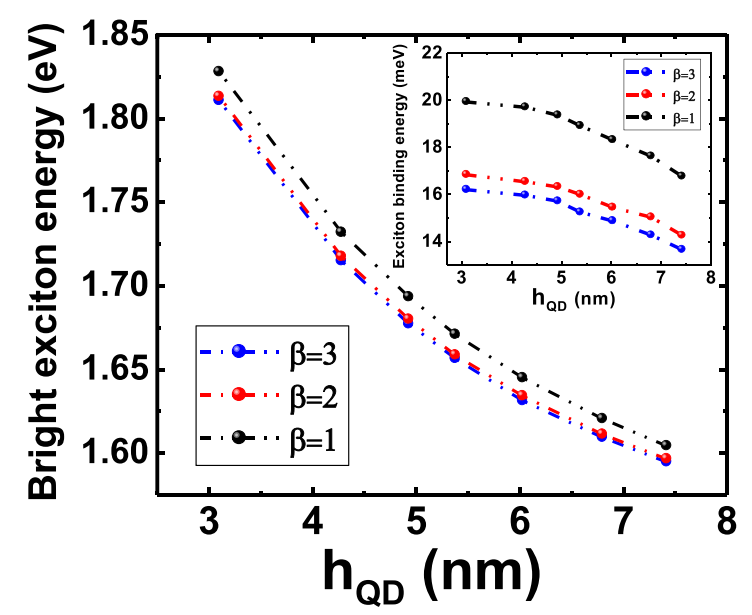

FIG. 2. Bright exciton energy versus $h_{\mathrm{QD}}$ for different anisotropy factors $\beta$. The inset shows the calculated values of the exciton binding energy as a function of $h_{\mathrm{QD}}$.

explored on numerous systems, such as elliptical QD [33,45], InAs/GaAs lens and disk QDs [46], InAs trianglelike QDs $[47,48]$. Recently, Zielinski [32,49] has studied InAs/InP QDs and clearly demonstrated that nanostructure shape anisotropy has a strong impact on the magnitudes of dark and bright exciton splittings.

\section{A. Quantum dot shape and confinement potential}

The shape of the GaAs QDs obtained by droplet epitaxy is modelled as in Refs. [36,37]. Two revolution ellipsoids with different centers and eccentricities allow us to define the shape contours of the GaAs QD. The nanohole shape is described by an ellipsoid cap [denoted $\mathrm{P}_{1}$ in Fig. 1(b)] and the upper surface of the GaAs QD after nanohole filling is also described by another ellipsoid cap [denoted $\mathrm{P}_{2}$ in Fig. 1(b)]. The main parameters are the QD thickness $h_{\mathrm{QD}}$, the semiaxes $r_{0 x}$ and $r_{0 y}$ of the hole opening, and the in-plane anisotropy parameter defined as $\beta=r_{0 x} / r_{0 y}$ (for details see Ref. [37]). Following the AFM images given in Ref. [37], we have fixed $r_{0 y}=30$ $\mathrm{nm}$, and varied $h_{\mathrm{QD}}$ and $\beta$ to reproduce emission energies and FSS. In this modelization to simulate the experimental data, the QD volume, defined as the volume between the two ellipsoid surfaces $P_{1}$ and $P_{2}$, increases with the anisotropy without significant change in the emission energy (see Fig. 2 and comments below). This is in agreement with AFM measurements [17] done on very similar QDs and showing that isotropic QDs are obtained for small volumes, while the anisotropy increases with larger volumes. $h_{\mathrm{QD}}$ is related to GaAs experimental flux; we have then considered $h_{\mathrm{QD}}=3.10,4.28,4.93,5.38,6.03$, 6.80 , and $7.42 \mathrm{~nm}$.

\section{B. Coulomb interaction}

Once $\left(E_{v}, \Psi_{n}^{v}\left(\mathbf{r}_{v}\right)\right)$ are calculated, we then estimate the Coulombic contribution to the exciton energy and reproduce the excitonic wave function. For this purpose, we have performed a variational calculation and considered the following trial function:

$$
\Phi\left(\mathbf{r}_{e}, \mathbf{r}_{h}\right)=\mathcal{N}(a, \lambda)\left[\exp -\frac{1}{a} \sqrt{\left(x_{e}-x_{h}\right)^{2}+\left(y_{e}-y_{h}\right)^{2}+\lambda^{2}\left(z_{e}-z_{h}\right)^{2}}\right] \Psi_{n}^{e}\left(\mathbf{r}_{e}\right) \Psi_{n}^{h}\left(\mathbf{r}_{h}\right)
$$

It is composed by the product of the single particle wave functions obtained previously and a modified hydrogenic wave function. This approach is commonly used for quantum well, wire, or dot [50,51]. $\mathcal{N}$ is a normalization factor and $\Psi_{n}^{\nu}\left(\mathbf{r}_{v}\right)(v=e, h)$ is the eigenfunction of the carrier $v . a$ and $\lambda$ represent the variational parameters, $a$ acts as an effective Bohr radius and $\lambda$ has been introduced to take into account the large anisotropy between the $\mathrm{O} z$ axis and the $x \mathrm{O} y$ plane $(\lambda$ improves the binding energy determination by $0.1-0.2 \mathrm{meV}$. No significant improvement has been observed by adding an extra parameter for the $x \mathrm{O} y$ anisotropy). Using the exciton Hamiltonian:

$$
\mathcal{H}_{T}=\mathcal{H}_{e}+\mathcal{H}_{h}-\frac{e^{2}}{4 \pi \epsilon_{0} \epsilon_{r}\left|\mathbf{r}_{e}-\mathbf{r}_{h}\right|}
$$

with $\epsilon_{0}$ the vacuum permittivity and $\epsilon_{r}$ the exciton dielectric constant (we have taken $\epsilon_{r}=12.53$ in the present numerical calculations), it is then possible to deduce the exciton energy $E_{X}$ by minimizing $\left\langle\mathcal{H}_{T}\right\rangle$ versus $a$ and $\lambda$. The exciton binding energy is defined as $E_{B}=\left(E_{g}+E_{e}+E_{h}\right)-E_{X}, E_{g}$ being the bulk band-gap energy.

Figure 2 presents the energy of the BE states for different anisotropy factors $\beta$, in the absence of electron-hole exchange energy, but including the direct Coulomb interaction. As expected, the exciton energy decreases as the QD thickness increases, leading to a reduction of the confinement. One can note that the exciton energy is mainly sensitive to the $z$-axis confinement (associated to the $h_{\mathrm{QD}}$ parameter) and much less to the ellipticity ( $\beta$ parameter). The exciton binding energy also decreases with QD thickness following a reduction of the electron and hole wave function overlap. However, a small impact of the $\beta$ value can be observed on the exciton binding energy $E_{B}$ (see the inset of Fig. 2) which is related to the increase of the confinement along the $x$ axis when $\beta$ decreases. The $x y$ confinement in our QD increases $E_{B}$ by a factor of two compared to GaAs/AlGaAs quantum wells with a thickness in the 3-8 $\mathrm{nm}$ range and $E_{B} \simeq 9 \mathrm{meV}$ [52] and we also underline that $E_{B}$ is varying slowly with $h_{\mathrm{QD}}$ in agreement with the quantum wells of Ref. [52]. In the following, we will use this exciton wave function $\Phi\left(\mathbf{r}_{e}, \mathbf{r}_{h}\right)$ to estimate the e-h LR and SR exchange interaction (EI) contributions.

\section{Basis function for holes}

To explore the effect of the valence band mixing on the e-h EI, we use the six-band $\mathbf{k} \cdot \mathbf{p}$ hole Hamiltonian, for which 
the notations are given in Appendix B. Here, we investigate the role of the e-h EI on the excitonic properties by explicitly taking into account the contributions of $\mathrm{HH}, \mathrm{LH}$, and split-off (SO) bands while reducing the Hilbert space. For this purpose, $\mathrm{HH}$ band being energetically lower than LH and SO bands, we carry out the Löwdin renormalization $[53,54]$ to get the new basis states for holes, namely the HH-like states, which are a mixture of the heavy- with the light- and SO-hole states. We label these hole ground states as $|\widetilde{3 / 2}\rangle$ and $|-\widetilde{3 / 2}\rangle$. With the perturbation formalism applying at second order, we can write the HH-like states as

$$
\begin{aligned}
\widetilde{3 / 2\rangle}= & \eta|3 / 2,3 / 2\rangle+\frac{\mathfrak{C}^{*}}{\left(E_{\ell h}-E_{h h}\right)}|3 / 2,-1 / 2\rangle \\
& -\frac{\sqrt{2} \mathfrak{C}^{*}}{\left(E_{s o}-E_{h h}\right)}|1 / 2,-1 / 2\rangle \\
\widetilde{-3 / 2\rangle}= & \eta|3 / 2,-3 / 2\rangle+\frac{\mathfrak{C}}{\left(E_{\ell h}-E_{h h}\right)}|3 / 2,1 / 2\rangle \\
& +\frac{\sqrt{2} \mathfrak{C}}{\left(E_{s o}-E_{h h}\right)}|1 / 2,1 / 2\rangle,
\end{aligned}
$$

where $\left|J^{h}, J_{z}^{h}\right\rangle$ are the periodic parts of the Bloch states of bulk bands, more precisely the $p$-like valence bands. Here $|3 / 2, \pm 3 / 2\rangle,|3 / 2, \pm 1 / 2\rangle$, and $|1 / 2, \pm 1 / 2\rangle$ correspond to $\mathrm{HH}, \mathrm{LH}$, and SO states, respectively. The parameter $\eta$ verifies

$$
\eta=1-\left(\frac{1}{\left(E_{\ell h}-E_{h h}\right)^{2}}+\frac{2}{\left(E_{s o}-E_{h h}\right)^{2}}\right)|\mathfrak{C}|^{2} .
$$

The term without $\mathfrak{C}$ in the last equation is derived from the $\mathrm{HH}$ band while the terms with $\mathfrak{C}$ are derived from the HH-LH and HH-SO interactions. Note that, employing the perturbation theory at first order, we get $\eta=1$. The $\mathfrak{C}$ expression is given explicitly in Appendix B [Eq. (B3)]. $E_{h h}, E_{\ell h}$, and $E_{s o}$ denote the energy of the HH, LH and SO hole states, respectively, which are estimated within the effective mass approximation and the BenDaniel Duke Hamiltonian [55] following the theoretical model developed in Ref. [37]. The $\mid \widetilde{3 / 2\rangle}(|\widetilde{-3 / 2}\rangle)$ state is divided into two parts: a major component which is $|3 / 2,3 / 2\rangle(|3 / 2,-3 / 2\rangle)$ and additional components that admixtures the HH, LH, and SO bands. Due to this bandmixing effect, we refer to the $\mathrm{BE}$ states by $\left|C_{-}, \widetilde{3 / 2}\right\rangle$ and $\left|C_{+}, \widetilde{-3 / 2}\right\rangle$, and the DE states as $\left|C_{+}, \widetilde{3 / 2}\right\rangle$ and $\left|C_{-}, \widetilde{-3 / 2}\right\rangle$. Having determined the expressions of this excitonic quartet, we are now able to calculate the $4 \times 4$ matrix representation of the e-h EI by taking into account the two conduction bands and six valence bands (HH, LH, SO) each doubly degenerate due to spin.

\section{Electron-hole exchange interaction}

We begin to derive the matrix representation of the LR EI, $\mathcal{H}_{\text {exch }}^{\mathrm{LR}}$, by adopting the following basic equation [40]

$$
\begin{aligned}
\mathcal{H}_{\mathrm{exch}}^{\mathrm{LR}}= & \frac{1}{(2 \pi)^{3}} \int d \mathbf{q}\left(\frac{e^{2}}{\epsilon_{0} \epsilon_{X}} \frac{1}{q^{2}}\right) \sum_{i, j} \underset{\substack{\mathfrak{K}^{\prime} \mathcal{K}^{\prime} m \\
\mathfrak{L}^{\prime} m}}{i j} q_{i} q_{j} \\
& \times \exp \left[i \mathbf{q} .\left(\mathbf{r}_{e}-\mathbf{r}_{e}^{\prime}\right)\right] \delta\left(\mathbf{r}_{e}-\mathbf{r}_{h}\right) \delta\left(\mathbf{r}_{e}^{\prime}-\mathbf{r}_{h}^{\prime}\right),
\end{aligned}
$$

where $m, m^{\prime}\left(n, n^{\prime}\right)$ indicate the Bloch states of the electron in the conduction band (the hole in the valence band), $\epsilon_{X}$ being the high-frequency (at the exciton resonance) dielectric constant, and $\mathcal{K}$ is the time-reversal operator. $\mathbf{q}=\left(q_{x}, q_{y}, q_{z}\right)$ denotes the wave vector in the reciprocal space and $q^{2}=q_{x}^{2}+q_{y}^{2}+q_{z}^{2}$. The explicit form of the matrix representation $\underset{\mathfrak{Q}_{m^{\prime} n^{\prime}}}{\mathfrak{m}^{\prime}}(\mathbf{q})=\sum_{i, j} \mathfrak{Q}_{\mathcal{K}_{n^{\prime} m} \mathcal{K}_{n}}^{i j} q_{i} q_{j}$ is given in Appendix C. Adopting equation (C3) and using the e-h states $\left\{\left|C_{-}, \widetilde{3 / 2}\right\rangle,\left|C_{+}, \widetilde{-3 / 2}\right\rangle,\left|C_{+}, \widetilde{3 / 2}\right\rangle,\left|C_{-}, \widetilde{-3 / 2}\right\rangle\right\}$ as basis, the following matrix representation is obtained:

$$
\mathcal{H}_{\text {exch }}^{\mathrm{LR}}=\left(\begin{array}{cccc}
\mathcal{H}_{d b}^{\mathrm{LR}} & \mathcal{H}_{\text {odb }}^{\mathrm{LR}} & 0 & 0 \\
\text { c.c. } & \mathcal{H}_{d b}^{\mathrm{LR}} & 0 & 0 \\
0 & 0 & \mathcal{H}_{d d}^{\mathrm{LR}} & \mathcal{H}_{\mathrm{odd}}^{\mathrm{LR}} \\
0 & 0 & \text { c.c. } & \mathcal{H}_{d d}^{\mathrm{LR}}
\end{array}\right),
$$

where c.c. denotes the complex conjugate. The matrix elements of $\mathcal{H}_{\text {exch }}^{\mathrm{LR}}$ are given by:

$$
\mathcal{H}_{\alpha}^{\mathrm{LR}}=\int d \mathbf{q} \mathfrak{L}_{\alpha} \mathcal{V}_{\mathbf{q}} \exp i \mathbf{q} .\left(\mathbf{r}_{e}-\mathbf{r}_{e}^{\prime}\right) \delta\left(\mathbf{r}_{e}-\mathbf{r}_{h}\right) \delta\left(\mathbf{r}_{e}^{\prime}-\mathbf{r}_{h}^{\prime}\right)
$$

in which $\alpha$ denotes the bright excitons contributions (db, odb) as well as the ones of the dark excitons (dd, odd), hence $\alpha=d b, o d b, d d, o d d ; \mathcal{V}_{\mathbf{q}}=\left[1 /(2 \pi)^{3}\right]\left(e^{2} / \epsilon_{0} \epsilon_{X}\right)\left(1 / q^{2}\right)$ is the Fourier transform of the Coulomb potential. We have taken $\epsilon_{X}=12.53$ in our calculations. The diagonal terms, $\left(\mathfrak{L}_{d b}, \mathfrak{L}_{d d}\right)$, and the off-diagonal ones, $\left(\mathfrak{L}_{\text {odb }}, \mathfrak{L}_{\text {odd }}\right)$, are expressed as

$$
\begin{aligned}
\mathfrak{L}_{d b}= & A \eta^{2} K^{2}-\frac{K_{-}^{2}}{\sqrt{3}}\left[\frac{A}{\left(E_{\ell h}-E_{h h}\right)}+\frac{B}{\left(E_{s o}-E_{h h}\right)}\right] \eta \mathfrak{C}^{*} \\
& -\frac{K_{+}^{2}}{\sqrt{3}}\left[\frac{A}{\left(E_{\ell h}-E_{h h}\right)}+\frac{B}{\left(E_{s o}-E_{h h}\right)}\right] \eta \mathfrak{C}^{\mathfrak{s}} \\
& +\frac{K^{2}}{3}\left[\frac{A}{\left(E_{\ell h}-E_{h h}\right)^{2}}+\frac{2 B}{\left(E_{\ell h}-E_{h h}\right)\left(E_{s o}-E_{h h}\right)}\right. \\
& \left.+\frac{2 C}{\left(E_{s o}-E_{h h}\right)^{2}}\right]|\mathfrak{C}|^{2} \\
\mathfrak{L}_{o d b}= & A \eta^{2} K_{-}^{2}-\frac{2 K^{2}}{\sqrt{3}}\left[\frac{A}{\left(E_{\ell h}-E_{h h}\right)}+\frac{B}{\left(E_{s o}-E_{h h}\right)}\right] \eta \mathfrak{C}^{\mathfrak{C}} \\
& +\frac{K_{+}^{2}}{3}\left[\frac{A}{\left(E_{\ell h}-E_{h h}\right)^{2}}+\frac{2 B}{\left(E_{\ell h}-E_{h h}\right)\left(E_{s o}-E_{h h}\right)}\right. \\
& \left.+\frac{2 C}{\left(E_{s o}-E_{h h}\right)^{2}}\right] \mathfrak{C}^{2} \\
\mathfrak{L}_{d d}= & \frac{2}{3} q_{z}^{2}\left[\frac{2 A}{\left(E_{\ell h}-E_{h h}\right)^{2}}-\frac{2 B}{\left(E_{\ell h}-E_{h h}\right)\left(E_{s o}-E_{h h}\right)}\right. \\
& \left.+\frac{C}{\left(E_{s o}-E_{h h}\right)^{2}}\right] \mid \mathfrak{C}^{2} \\
\mathfrak{L}_{\mathrm{odd}}= & -\frac{2}{3} q_{z}^{2}\left[\frac{2 A}{\left(E_{\ell h}-E_{h h}\right)^{2}}-\frac{2 B}{\left(E_{\ell h}-E_{h h}\right)\left(E_{s o}-E_{h h}\right)}\right. \\
& \left.+\frac{C}{\left(E_{s o}-E_{h h}\right)^{2}}\right] \mathfrak{C}^{2} .
\end{aligned}
$$

Note that, in the dark states subspace, nonzero LR terms only appear at the second order. $K^{2}=\left(q_{x}^{2}+q_{y}^{2}\right), K_{ \pm}=\left(q_{x} \pm i q_{y}\right)$, 
$A=\left(\hbar^{2} / 4 m_{0}\right)\left(E_{P} / E_{g}^{2}\right), \quad B=\left(\hbar^{2} / 2 m_{0}\right)\left(E_{P} / E_{g}\left(E_{g}+\Delta_{s o}\right)\right)$, and $C=\left(\hbar^{2} / 2 m_{0}\right)\left(E_{P} /\left(E_{g}+\Delta_{s o}\right)^{2}\right) . E_{P}$ is the Kane energy, $m_{0}$ is the free electron mass, and $\Delta_{s o}$ is the spin-orbit coupling parameter. Taking $\left(E_{g}+\Delta_{s o}\right)$ approximately equal to $E_{g}$ in the relationships of $B$ and $C$, we get $C \simeq B \simeq 2 A$, and this has been adopted systematically in our calculations. The $\mathfrak{C}$ quadratic terms in Eqs. (8) and (9) have not been taken into account in the present modelization.

In addition to the LR EI addressed above, the SR EI also contributes to describe the exciton fine structure. For this purpose, we directly derive the matrix representation of the SR EI, $\mathcal{H}_{\text {exch }}^{\mathrm{SR}}$, by adopting the Eq. (C4) of Appendix C. In the basis $\left\{\left|C_{-}, \widetilde{3 / 2}\right\rangle,\left|C_{+}, \widetilde{-3 / 2}\right\rangle,\left|C_{+}, \widetilde{3 / 2}\right\rangle,\left|C_{-}, \widetilde{-3 / 2}\right\rangle\right\}$, the $\mathcal{H}_{\text {exch }}^{\mathrm{SR}}$ matrix takes the form

$$
\mathcal{H}_{\mathrm{exch}}^{\mathrm{SR}}=\left(\begin{array}{cccc}
\mathcal{H}_{d b}^{\mathrm{SR}} & \mathcal{H}_{\text {odb }}^{\mathrm{SR}} & 0 & 0 \\
\mathrm{c} . \mathrm{c} . & \mathcal{H}_{d b}^{\mathrm{SR}} & 0 & 0 \\
0 & 0 & \mathcal{H}_{d d}^{\mathrm{SR}} & \mathcal{H}_{\text {odd }}^{\mathrm{SR}} \\
0 & 0 & \text { c.c. } & \mathcal{H}_{d d}^{\mathrm{SR}}
\end{array}\right) .
$$

One expresses the nonzero matrix elements of $\mathcal{H}_{\mathrm{exch}}^{\mathrm{SR}}$ as

$$
\mathcal{H}_{\alpha}^{\mathrm{SR}}=D \mathcal{S}_{\alpha} \delta\left(\mathbf{r}_{e}-\mathbf{r}_{h}\right) \delta\left(\mathbf{r}_{e}-\mathbf{r}_{e}^{\prime}\right) \delta\left(\mathbf{r}_{h}-\mathbf{r}_{h}^{\prime}\right),
$$

$\alpha$ being $\alpha=d b$, odb, $d d$, odd. The SR exchange constant is defined as $D=(3 / 4) \pi a_{X}^{3} \Delta_{\mathrm{SR}}$, where $a_{X}$ is the bulk Bohr radius $\left(a_{X}=\left[4 \pi \epsilon_{0} \epsilon_{X} \mu / e^{2}\right]=14.5 \mathrm{~nm}\right.$, in which $\mu^{-1}=$ $\left[m_{e}^{-1}+\gamma_{1} m_{0}^{-1}\right]$ ( $\gamma_{1}$ being the Luttinger parameter) and the electron mass is taken as $m_{e}=0.067 m_{0}$ [56]) and $\Delta_{\mathrm{SR}}$ is the analytical exchange constant as defined in Ref. [57]. From now, $D$ has been fixed at $57.8 \mathrm{meV} \mathrm{nm}^{3}$, in agreement with the experimental values obtained in GaAs bulk [57] and GaAs/AlGaAs quantum wells [58]. The $\mathcal{S}_{\alpha}$ expressions in Eq. (13) can approximately be written:

$$
\begin{gathered}
\mathcal{S}_{d b}=\eta^{2}+\frac{1}{3}\left[\frac{1}{\left(E_{\ell h}-E_{h h}\right)}+\frac{2}{\left(E_{s o}-E_{h h}\right)}\right]^{2}|\mathfrak{C}|^{2} \\
\mathcal{S}_{o d b}=-\frac{2}{\sqrt{3}}\left[\frac{1}{\left(E_{\ell h}-E_{h h}\right)}+\frac{2}{\left(E_{s o}-E_{h h}\right)}\right] \eta \mathfrak{C}^{2} \\
\mathcal{S}_{d d}=\frac{2}{3}\left[\frac{1}{\left(E_{\ell h}-E_{h h}\right)}-\frac{1}{\left(E_{s o}-E_{h h}\right)}\right]^{2}|\mathfrak{C}|^{2} \\
\mathcal{S}_{\text {odd }}=-\frac{2}{3}\left[\frac{1}{\left(E_{\ell h}-E_{h h}\right)}-\frac{1}{\left(E_{s o}-E_{h h}\right)}\right]^{2} \mathfrak{C}^{2} .
\end{gathered}
$$

The term without $\mathfrak{C}$ in expressions (8)-(11) and (14)-(17) originates from the $\mathrm{HH}$ band whereas the terms with $\mathfrak{C}$ in these expressions are associated to the (HH-LH) and (HH-SO) couplings. Putting together $\mathcal{H}_{\text {exch }}^{\mathrm{LR}}$ and $\mathcal{H}_{\text {exch }}^{\mathrm{SR}}$, we deduce an exchange Hamiltonian which has the same shape as the one obtained in $C_{2 v}$ symmetry [59]. From the $\left(\mathcal{H}_{\mathrm{exch}}^{\mathrm{LR}}+\mathcal{H}_{\mathrm{exch}}^{\mathrm{SR}}\right)$ matrix form, one can see that the excitonic states are split into four energy sublevels as a result of the exchange interaction between electron and hole spins [see Fig. 1(a)]. The quadruplet is splits into two doublets, bright and dark doublets, with the bright one situated above the dark one. The off-diagonal matrix elements $\left(\mathcal{H}_{\text {odb }}^{\mathrm{LR}}+\mathcal{H}_{\text {odb }}^{\mathrm{SR}}\right)$ and $\left(\mathcal{H}_{\text {odd }}^{\mathrm{LR}}+\mathcal{H}_{\text {odd }}^{\mathrm{SR}}\right)$ describe the BB and DD energy splitting, respectively. Note that, due to the $\mathfrak{C}^{2}$ dependence of $\mathcal{L}_{\text {odd }}$ and $\mathcal{S}_{\text {odd }}$, the perturbation theory applied at first order does not provide the dark exciton doublet splitting and the second-order perturbation theory is

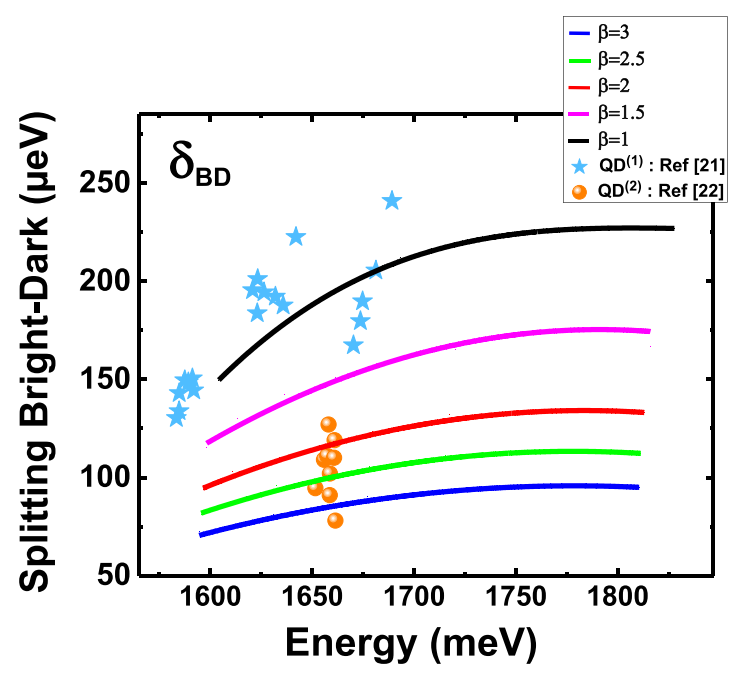

FIG. 3. Measured bright-dark splittings [21,22] and calculated values as a function of the bright exciton energy for different anisotropy factor $\beta$.

then needed to calculate the DD splitting. For the DB exciton splitting, all the matrix elements of $\left(\mathcal{H}_{\text {exch }}^{\mathrm{LR}}+\mathcal{H}_{\mathrm{exch}}^{\mathrm{SR}}\right)$ contribute to the energy difference between the lowest $\mathrm{BE}$ state and the uppermost DE state.

\section{RESULTS}

Few groups are able to grow strain-free QDs in the nanohole discussed in this paper, but recent progress has permitted to evidence the FSS, including either bright or dark states [21,22,37]. DE states were revealed in QDs placed near a cleaved edge [21] or by applying an in-plane magnetic field [22]. In Ref. [21], the two dark states were visible, meanwhile only the upper dark state, $\left|\mathrm{DE}_{+}\right\rangle$, was brightened. Following the modelization described in the previous section, we have compared the published experimental results with the FSS deduced from the theory.

We first consider the splitting $\delta_{\mathrm{BD}}$ between the lowest energy $\mathrm{BE}$ state, $\left|\mathrm{BE}_{-}\right\rangle$, and the highest energy $\mathrm{DE}$ state, $\left|\mathrm{DE}_{+}\right\rangle$, following the convention of Refs. [21,60]. Two different QD series are considered: In Ref. [21], the QDs are grown in round nanoholes, while in Ref. [22], the nanoholes are highly anisotropic. Theoretical curves and data of Refs. [21,22] are represented versus exciton energy in Fig. 3. The theoretical $\delta_{\mathrm{BD}}$ curves are given for isotropic $(\beta=1)$ and anisotropic $(\beta>1)$ nanoholes. For isotropic QDs [21], within the experimental dispersion, the splitting $\delta_{\mathrm{BD}}$ is well reproduced with an in-plane anisotropy parameter close to unity, in very good agreement with the nanohole shape. All the data being at higher energy than the $\beta=1.5$ curve, one can explain the $\delta_{\mathrm{BD}}$ dispersion by a small dispersion in the in-plane shape and in the $\beta$ parameter $(\beta<1.5)$. For the highly anisotropic nanoholes [22], the average of the $\delta_{\mathrm{BD}}$ values is close to the $\beta=2.5$ curve. The dispersion in the $\delta_{\mathrm{BD}}$ values might be explained by a dispersion in $\beta(=2.5 \pm 0.7)$ in these very anisotropic QDs. The energy emission is mainly fixed by the QD thickness $h_{\mathrm{QD}}$, while $\delta_{\mathrm{BD}}$ will strongly vary with $\beta$. Note 


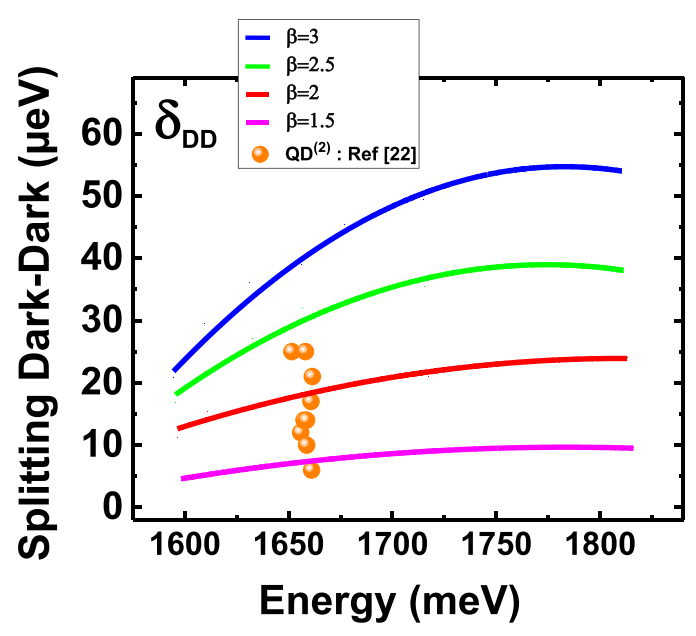

FIG. 4. Measured dark-dark splittings [22] and calculated values as a function of the bright exciton energy for different anisotropy factor $\beta$.

that the LR contribution in $\delta_{\mathrm{BD}}$ varies from about $70 \%$ to $10 \%$ when $\beta$ varies from 1 to 3 .

Being able to model the BD splitting by correlating $\delta_{\mathrm{BD}}$ with emission energy and shape, we have then considered the splitting $\delta_{\mathrm{DD}}$ between the two DE states, rarely observed for such a QD family. This doublet is clearly visible in Ref. [22], by applying in-plane magnetic field, while only one transition has been observed in QDs placed near a cleaved edged [21]. This splitting $\delta_{\mathrm{DD}}$ is small $(10-25 \mu \mathrm{eV})$ and depends on LR and SR second-order contributions (see the $\mathcal{L}_{\text {odd }}$ and $\mathcal{S}_{\text {odd }}$ expressions) which are related to the valence band mixing, particularly the HH-LH mixing. As already observed in other QDs, such as InAs/InP, the DD splitting is very sensitive to the HH-LH coupling [32]. The theoretical curves (see Fig. 4) are very sensitive to $\beta$ (the $\beta=1$ curve is not represented). For the same QD parameters as in Fig. 3, a good agreement is obtained between the average of $\delta_{\mathrm{DD}}$ experimental values and the $\beta \approx 2$ curve (see Fig. 4 ), a value very close to the one estimated from $\delta_{\mathrm{BD}}$ (Fig. 3). The experimental dispersion might be explained by a fluctuation in the anisotropy parameter and/or on the fluctuation HH-LH mixing values from one QD to another QD.

Finally, keeping the same parameters $\left(h_{\mathrm{QD}}, \beta\right)$ as for $\delta_{\mathrm{BD}}$ and $\delta_{\mathrm{DD}}$, we have calculated the splitting $\delta_{\mathrm{BB}}$ between the two BE states, commonly observed in QD. We have reported in Fig. 5 the experimental values of $\delta_{\mathrm{BB}}$ obtained on three series of GaAs/AlGaAs QD grown in nanoholes [21,22,37]: QDs of Refs. [22,37] are anisotropic, while those of Ref. [21] are mainly isotropic. Theoretical predictions, for $\beta$ values from 1.5 to 3 , are reported in order to take into account the small or large anisotropy observed by AFM/STM microscopy (the $\beta=1$ curve is not represented, $\delta_{\mathrm{BB}}$ being zero in our theoretical approach).

For the isotropic QDs, whose $\delta_{\mathrm{BD}}$ splittings are well reproduced for $\beta=1$ (see Fig. 3), one observes a theoretical underestimation of the $\delta_{\mathrm{BB}}$ splittings, the experimental values being 20 to $40 \mu \mathrm{eV}$ larger. Nonzero $\delta_{\mathrm{BB}}$ values have already been observed on isotropic droplet GaAs QDs; the smallest values observed in literature are given in Ref. [18]. For the

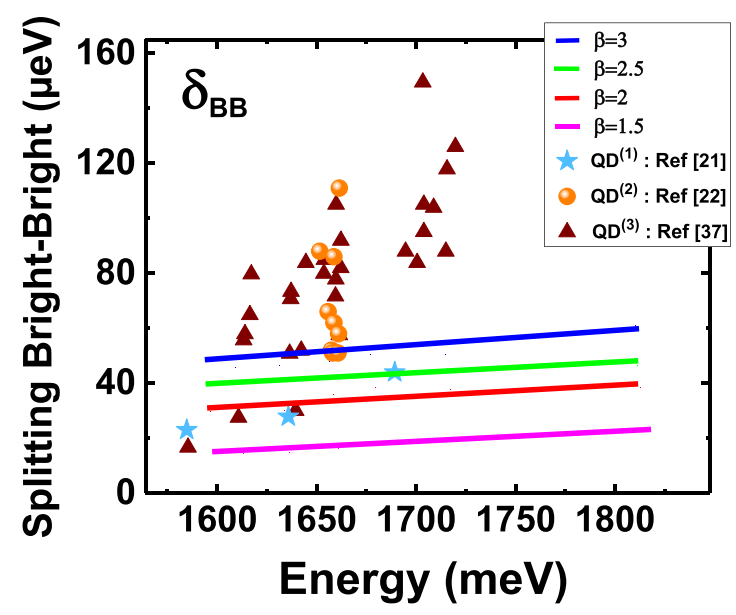

FIG. 5. Measured bright-bright splittings [21,22,37] and calculated values as a function of the bright exciton energy for different anisotropy factor $\beta$.

anisotropic QDs $(\beta=3)$, one also observes a discrepancy, the experimental values being up to $70 \mu \mathrm{eV}$ larger, for the highest emission energies. These discrepancies may be attributed to contributions not considered in our model and discussed in the literature:

(i) alloy fluctuations [61];

(ii) effects induced by atomic ordering at the interface as discussed for InGaAs/GaAs QDs [43];

(iii) effects due to the zinc-blende symmetry and nonequivalent [110] and [1-10] axes [42];

(iv) configuration interaction (or coupling with excited states) [32];

(v) $C_{2 v}$ broken symmetry in the QD shape [44].

The alloy effect (i) is weak, in the order of few $\mu \mathrm{eV}$ for $\mathrm{GaAs} / \mathrm{AlGaAs}$ or InAs/GaAs as discussed in Refs. [35,61]. The contribution of interface ordering (ii) is also strongly reduced by any alloy randomness at the interface. But other contributions can be larger (few tens of $\mu \mathrm{eV}$ ). For instance, from atomistic many-body theory, for highly isotropic droplet GaAs QDs, Luo and Zunger [42] have considered the intrinsic $C_{2 v}$ symmetry of the zinc-blende crystal [contribution (iii)] and evidenced splittings of several tens of $\mu \mathrm{eV}$ in the emission range considered.

Another contribution (iv) may come from a coupling between the $s$ ground states and excited states, currently considered on the CI calculations. As shown in Ref. [36], the CI contribution induces a small correction on the emission energy, and in the absence of anisotropy it has no effect on the FSS [62]. Nonetheless, very recent studies on InAs/InP QDs have evidenced the role of CI even for isotropic QDs [32]. For the particular system, InAs/InP elongated along the [1-10] axis with strain and a small volume $\left(h_{\mathrm{QD}}=3 \mathrm{~nm}\right.$ and $\left.\sqrt{r_{0 x} r_{0 y}}=20 \mathrm{~nm}\right)$, CI has a vanishing effect on $\delta_{\mathrm{DD}}$ for $\beta<4$ $\left(t=0-0.9\right.$ in Ref. [32]), while for $\beta=4, \delta_{\mathrm{BB}}$ and $\delta_{\mathrm{BD}}$ can be increased by up to $80 \%$ and $40 \%$, respectively.

Finally, for the QDs grown in anisotropic and certainly imperfect nanoholes, a small contribution could also come from the reduction of the $C_{2 v}$ symmetry as discussed by Zielinski et al. [44]. For example, Fuster et al. [63] have 
TABLE I. Summarized theoretical results of $\delta_{0}$ splitting (see Ref. [60]) for different kinds of GaAs QDs: nanocrystals (with spherical shape), interfacial QDs (with rectangular parallelepipedic shape), and GaAs droplet QDs (with shape defined in this work). The contributions of LR and SR are also given. The results of Refs. [64] and [65] are computed from empirical pseudopotential approach.

\begin{tabular}{|c|c|c|c|c|c|}
\hline \multicolumn{6}{|c|}{ GaAs nanocrystals $^{\mathrm{a}}$} \\
\hline Radius (nm) & Volume $\left(\mathrm{nm}^{3}\right)$ & $\mathrm{LR}(\mathrm{meV})$ & $\mathrm{SR}(\mathrm{meV})$ & $\delta_{0}(\mathrm{meV})$ & {$\left[\mathrm{LR} / \delta_{0}\right](\%)$} \\
\hline 1.9 & 28.73 & 4.1 & 2.3 & 6.4 & 64 \\
\hline 2 & 33.51 & 3.7 & 1.8 & 5.5 & 67.3 \\
\hline 2.5 & 65.45 & 2.4 & 1.2 & 3.6 & 66.7 \\
\hline 3 & 113.1 & 1.8 & 0.4 & 2.2 & 81.8 \\
\hline 4 & 268.1 & 1.1 & 0.2 & 1.3 & 84.6 \\
\hline \multicolumn{6}{|c|}{ Interface GaAs QDs ${ }^{\mathrm{b}}$} \\
\hline Size $(\mathrm{nm})$ & Volume $\left(\mathrm{nm}^{3}\right)$ & $\mathrm{LR}(\mu \mathrm{eV})$ & $\mathrm{SR}(\mu \mathrm{eV})$ & $\delta_{0}(\mu \mathrm{eV})$ & {$\left[L R / \delta_{0}\right](\%)$} \\
\hline $20 \times 20 \times 3.1$ & 1240 & 6 & 2.8 & 8.8 & 68.2 \\
\hline $40 \times 20 \times 3.1$ & 2480 & 5.2 & 2 & 7.2 & 72.2 \\
\hline $40 \times 40 \times 3.1$ & 4960 & 3.6 & 1.6 & 5.2 & 69.2 \\
\hline \multicolumn{6}{|c|}{ GaAs droplet QD (this work) } \\
\hline Size $(\mathrm{nm}) r_{0 x}=r_{0 y}=30 \mathrm{~nm} h_{\mathrm{QD}}$ & Volume $\left(\mathrm{nm}^{3}\right)$ & $\mathrm{LR}(\mu \mathrm{eV})$ & $\mathrm{SR}(\mu \mathrm{eV})$ & $\delta_{0}(\mu \mathrm{eV})$ & {$\left[L R / \delta_{0}\right](\%)$} \\
\hline 3.1 & 5843 & 154.7 & 72.3 & 227 & 68.2 \\
\hline 4.28 & 8068 & 149.8 & 70.7 & 220.5 & 67.9 \\
\hline 4.93 & 9293 & 142.2 & 69.9 & 212.1 & 67 \\
\hline 5.38 & 10141 & 133.3 & 65.7 & 199 & 67 \\
\hline 6.03 & 11366 & 122.1 & 61.5 & 183.6 & 66.5 \\
\hline 6.8 & 12818 & 111.3 & 55 & 166.3 & 66.9 \\
\hline 7.42 & 13986 & 99.3 & 50 & 149.3 & 66.5 \\
\hline
\end{tabular}

${ }^{\text {aRef. [64]. }}$

${ }^{\mathrm{b}}$ Ref. [65].

evidenced by AFM images an asymmetry of the hole profile along the [1-10] axis reducing the $C_{2 v}$ symmetry. One of the consequences of this symmetry reduction is the mixing of dark and bright states via nonzero terms in the off-diagonal dark and bright subspaces in the matrix describing the exchange Hamiltonian and resulting from the addition of matrices given by equations (6) and (12). Then, this symmetry reduction acts as an effective in-plane magnetic field.

In summary, for the isotropic QDs $(\beta=1)$, the contributions (iii) and (iv) could explain our underestimation of $\delta_{\mathrm{BB}}$, by 20 to $40 \mu \mathrm{eV}$. For anisotropic QDs $(\beta>1)$, contributions (iii), (iv), and (v) cannot be excluded and taking into account these contributions could improve the agreement theory experiment. For the anisotropic QDs, one may expect an increasing CI correction when the anisotropy increases (the influence of the thickness having to be explored). Modelization of the symmetry breaking would need to consider more complex shapes than the one given in Fig. 1 (as including a truncated facet, tilting or shifting the ellipsoid $\mathrm{P}_{2} \ldots$... Taking into account this symmetry breaking increases the numerical demand and is beyond the scope of this paper. Moreover, authors of Ref. [22] did not observe luminescence at zero magnetic field coming from dark states in the limit of sensitivity of their experiment, meaning that $C_{2 v}$ symmetry breaking, in this case, is not very important. We underline also that the discussed contributions impact especially the excitonic splitting $\delta_{\mathrm{BB}}$, in agreement with the results shown in Figs. 3-5.

Figures 6(a)-6(c) show the values of the BD, BB, and DD splittings and their respective LR and SR contributions as a function of the anisotropy parameter $\beta$ for a size of $h_{\mathrm{QD}}=$ $4.93 \mathrm{~nm}$ in the middle of the explored size domain. The LR contribution represents almost $68 \%$ to $5 \%$ of the total $\mathrm{BD}$ splitting and around $70 \%$ of the total BB splitting for $\beta \neq 1$ and constitutes, once again for $\beta \neq 1$, the greater part (86\%) of the DD splitting. We underline that SR contribution to BB splitting is negative. The trend is that the LR contribution weight to the total BD splitting decreases as the anisotropy of the droplet QD increases meanwhile the LR contribution weight to BB and DD splittings remains almost constant. We show that LR contribution is essential to well describe not only the BB and DD splittings but also the BD splitting. Finally, we point out that on the frame of hypothesis of this work, i.e., on the frame of $C_{2 v}$ symmetry for QDs, the LR contribution plays a major role on the DD splitting evaluation.

Table I summarizes theoretical results of BD splitting and contributions of SR and LR for different kinds of GaAs QDs [64,65]. Only symmetric QDs are considered. In order to compare with other authors, we use $\delta_{0}$ (see Ref. [60]), but for symmetric QDs the difference between $\delta_{\mathrm{BD}}$ and $\delta_{0}$ is very small. We find that $\delta_{0}$ takes very different values covering several orders of magnitude from 0.01 to $10 \mathrm{meV}$. The smallest values are found for thickness fluctuations QDs and the largest ones for GaAs nanocrystals. The LR contribution is very important for all the compared QDs, representing in general $64-85 \%$ of the total BD splitting. For a given kind of $\mathrm{QD}, \delta_{0}$ increases with decreasing volume or increasing quantum confinement. Very different values of $\delta_{0}$ are found for QDs having similar volumes. These results highlight the fact that the relative importance of LR and SR part in the BD splitting and their dependences in size, shape, or confinement potential are complex questions that cannot be addressed with simplified models. 
(a)

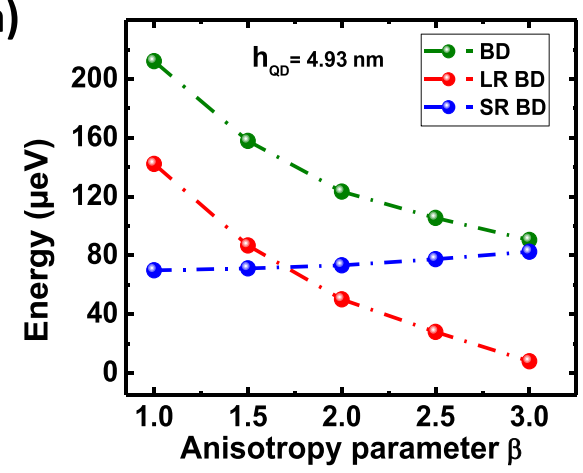

(b)

(c)
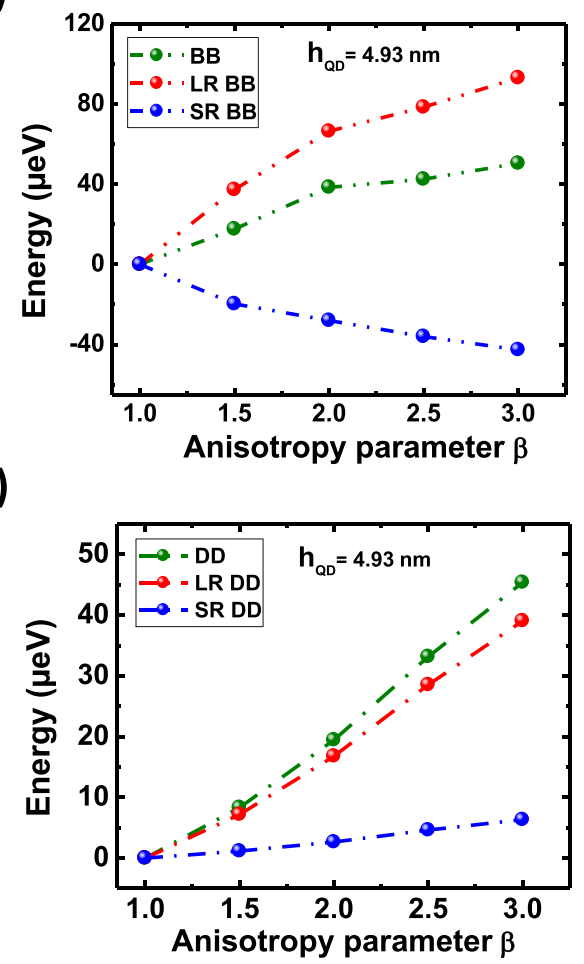

FIG. 6. (a) BD splitting and the respective LR and SR contributions, versus the anisotropy parameter $\beta$. (b) BB splitting and the respective LR and SR contributions. (c) LR and SR contributions in DD splitting versus the anisotropy parameter $\beta$. All the splittings have been obtained for $h_{\mathrm{QD}}=4.93 \mathrm{~nm}$.

\section{CONCLUSIONS}

We studied the full excitonic fine structure of $\mathrm{GaAs} / \mathrm{AlGaAs}$ QDs, and the three splittings involved $\delta_{\mathrm{BB}}$, $\delta_{\mathrm{BD}}$, and $\delta_{\mathrm{DD}}$. We have taken into consideration nanohole shape, Coulomb interaction, LR and SR coupling, including the first and the second-order contribution when necessary. Following the shape anisotropy deduced from AFM images, we have reproduced with the same QD parameters either $\delta_{\mathrm{BD}}$ and $\delta_{\mathrm{DD}}$. The dark-dark exciton splitting $\delta_{\mathrm{DD}}$, never calculated in such strain-free GaAs/AlGaAs QD, can only be reproduced when the second order long-range and short-range contributions are considered, including then the valence band mixing. The difference between our calculations and the experimental $\delta_{\mathrm{BB}}$ splittings may evidence the role of extra contributions not taken into account in our model: an intrinsic effect related to the nonequivalent [110] and [1-10] axes in zinc-blende crystal [42] and/or the configuration interaction contribution, particularly sensitive to the QD anisotropy [32]. The QD model and calculations developed for strain-free QDs synthesized by droplet epitaxy is validated by the very good agreement obtained between experiment and theory for both splittings, $\delta_{\mathrm{DD}}$ and $\delta_{\mathrm{BD}}$, while taking into account the observed nanohole shape. Future modelization, including intrinsic contributions, such as configuration interaction, or extrinsic contributions, such as $\mathrm{C}_{2 v}$ shape symmetry breaking, should lead to an even more accurate modelization of the nanostructures (photon-source and quantum bits) based on droplet epitaxied QD.

\section{ACKNOWLEDGMENTS}

We gratefully acknowledge valuable discussions and input from P. Atkinson and O. G. Schmidt. H.M., S.B.R., and K.B. thank the financial support of Tunisian Ministry of Higher Education and Scientific Research. H.M. was also supported by the Cluster of Excellence MATISSE led by Sorbonne University.

\section{APPENDIX A: CONFINED STATES}

We calculate the charge carrier states in the framework of the effective mass approximation. The Hamiltonian describing a single carrier [electron (e) or hole (h)] is given by

$$
\mathcal{H}_{v}=\mathcal{T}_{v}\left(\mathbf{r}_{v}\right)+\mathcal{V}_{v}\left(\mathbf{r}_{v}\right)
$$

in which $v=e, h, \mathcal{V}_{v}$ is the carrier confinement potential, and $\mathcal{T}_{v}$ denotes the carrier kinetic operator. Taking into account the cylindrical symmetry breaking, we can decompose the kinetic energy operator into three components: $\mathcal{T}_{v}=\mathcal{T}_{v}^{z}+\mathcal{T}_{v}^{\rho}+\mathcal{T}_{v}^{\theta}$, with

$$
\begin{aligned}
& \mathcal{T}_{v}^{z}=-\frac{\hbar^{2}}{2}\left[\frac{\partial}{\partial z} \frac{1}{m_{v}^{z}} \frac{\partial}{\partial z}\right] \\
& \mathcal{T}_{v}^{\rho}=-\frac{\hbar^{2}}{2}\left[\frac{1}{\rho} \frac{\partial}{\partial \rho} \frac{1}{m_{v}^{\rho}} \frac{\partial}{\partial \rho}\right] \\
& \mathcal{T}_{v}^{\theta}=-\frac{\hbar^{2}}{2}\left[\frac{1}{\rho^{2}} \frac{\partial}{\partial \theta} \frac{1}{m_{v}^{\rho}} \frac{\partial}{\partial \theta}\right],
\end{aligned}
$$

where $(\rho, \theta, z)$ denotes cylindrical coordinates. $m_{v}^{z}$ and $m_{v}^{\rho}$ are the carriers effective masses by $z$ and $\rho$ directions, respectively.

The eigenfunctions of the Hamiltonian are expanded in a Fourier-Bessel finite series as $\Psi_{n}^{\nu}\left(\mathbf{r}_{v}\right)=$ $\sum_{\ell>0, m>0}^{\ell_{\max }, m_{\max }} C_{\ell m}^{n} \phi_{\ell, m}^{n}\left(\rho_{\nu}, \theta_{\nu}, z_{\nu}\right)$, where $C_{\ell m}^{n}$ are the basis coefficients. The $\phi_{\ell, m}^{n}\left(\rho_{\nu}, \theta_{\nu}, z_{\nu}\right)$ functions correspond to the eigenbasis of a large cylinder having $\mathcal{Z}_{C}$ as a height and $\mathcal{R}_{C}(\theta)=\left[\sqrt{\left(\cos ^{2} \theta\right) / A^{2}+\left(\sin ^{2} \theta\right) / B^{2}}\right]^{-1}$ as an elliptical base radius. $A$ and $B$ denote the dimensions along $x$ and $y$ directions of the elongated cylinder, respectively. The normalization condition of the carriers wave functions leads to the condition $A / B=\beta=2.5$. The $z$-axis origin is taken at the midpoint of the cylinder. For the $s$ state, we take the following eigenbasis: $\phi_{\ell, m}^{0}\left(\rho_{\nu}, \theta_{\nu}, z_{\nu}\right)=\alpha_{\ell}^{0} J_{0}\left(\lambda_{0}^{\ell} \rho_{\nu} / \mathcal{R}_{C}(\theta)\right) \sin \left(\pi m z_{\nu} / \mathcal{Z}_{C}\right), \quad$ in which $\lambda_{0}^{\ell}$ is the $\ell$ th root of the $J_{0}$ Bessel function and $\alpha_{\ell}^{0}$ are the normalization constants. 


\section{APPENDIX B: SIX-BAND k • p HOLE HAMILTONIAN}

We denote the hole Bloch states as

$$
\begin{aligned}
|3 / 2,3 / 2\rangle & =-\frac{1}{\sqrt{2}}|(X+i Y) \uparrow\rangle \\
|3 / 2,1 / 2\rangle & =-\frac{1}{\sqrt{6}}|[(X+i Y) \downarrow-2 Z \uparrow]\rangle \\
|3 / 2,-1 / 2\rangle & =\frac{1}{\sqrt{6}}|[(X-i Y) \uparrow+2 Z \downarrow]\rangle \\
|3 / 2,-3 / 2\rangle & \left.\left.=\frac{1}{\sqrt{2}}||(X-i Y) \downarrow\right\rangle\right\rangle \\
|1 / 2,1 / 2\rangle & =-\frac{1}{\sqrt{3}}|[(X+i Y) \downarrow+Z \uparrow]\rangle \\
|1 / 2,-1 / 2\rangle & =-\frac{1}{\sqrt{3}}|[(X-i Y) \uparrow-Z \downarrow]\rangle,
\end{aligned}
$$

where $\uparrow(\downarrow)$ denotes the spin-up (spin-down) states and $|X\rangle,|Y\rangle,|Z\rangle$ represent the components of the $p$-like valence-band Bloch wave functions (derived from atomic $p_{x}, p_{y}, p_{z}$ orbitals).

In the $\{|3 / 2,3 / 2\rangle,|3 / 2,1 / 2\rangle,|3 / 2,-1 / 2\rangle,|3 / 2,-3 / 2\rangle,|1 / 2,1 / 2\rangle,|1 / 2,-1 / 2\rangle\}$ basis, the six-band hole Hamiltonian takes the following expression:

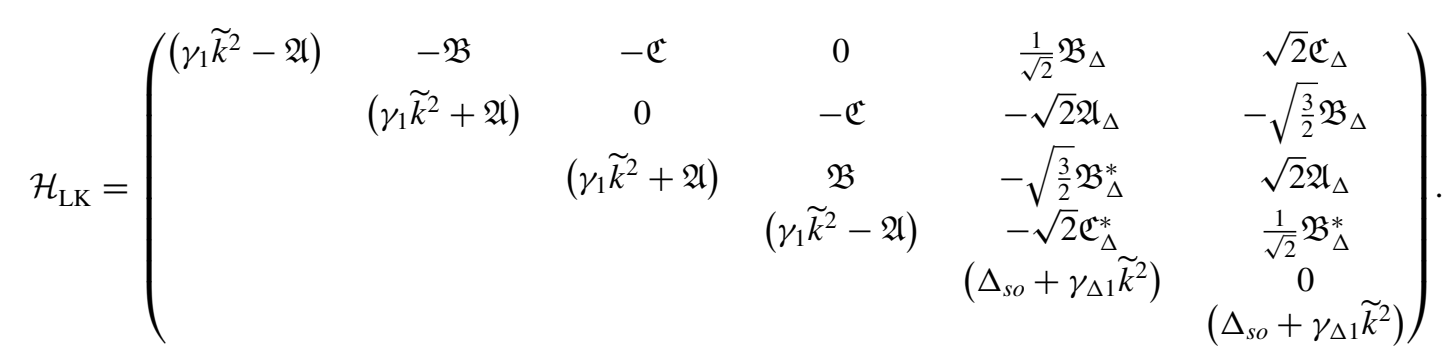

The other half of the matrix is obtained by taking the Hermitian conjugate. The nonzero elements of the matrix $\mathcal{H}_{\mathrm{LK}}$, namely $(\mathfrak{A}, \mathfrak{B}, \mathfrak{C})$, read as

$$
\begin{aligned}
\mathfrak{A} & =\gamma_{2}\left(2 \widetilde{k}_{z}^{2}-\widetilde{k}_{\rho}^{2}\right) \\
\mathfrak{B} & =2 \sqrt{3} \gamma_{3} \tilde{k}_{z} \tilde{k}_{-} \\
\mathfrak{C} & =\sqrt{3}\left[\gamma_{2}\left(\widetilde{k}_{x}^{2}-\widetilde{k}_{y}^{2}\right)-2 i \gamma_{3} \widetilde{k}_{x} \tilde{k}_{y}\right]
\end{aligned}
$$

in which $\widetilde{k}^{2}=\left(\hbar^{2} / 2 m_{0}\right) k^{2}, \widetilde{k}_{\rho}^{2}=\left(\widetilde{k}_{x}^{2}+\widetilde{k}_{y}^{2}\right)$, and $\widetilde{k}_{ \pm}=\left(\widetilde{k}_{x} \pm i \widetilde{k}_{y}\right)$.

Formally, this $\mathcal{H}_{\mathrm{LK}}$ Hamiltonian has the same structure as the Luttinger-Kohn Hamiltonian [54]. ( $\left.\mathfrak{A}_{\Delta}, \mathfrak{B}_{\Delta}, \mathfrak{C}_{\Delta}\right)$ take similar expressions to $(\mathfrak{A}, \mathfrak{B}, \mathfrak{C})$ in which the Luttinger parameters, $\left(\gamma_{1}, \gamma_{2}, \gamma_{3}\right)$, are substituted by the Luttinger-like parameters [66], $\left(\gamma_{\Delta 1}, \gamma_{\Delta 2}, \gamma_{\Delta_{3}}\right)$. Adopting the 14-band $\mathbf{k} \cdot \mathbf{p}$ model [67] which includes the $p$-like valence bands and $s$ - and $p$-like conduction bands, one can obtain:

$$
\begin{aligned}
& \gamma_{\Delta 1}=\gamma_{1}-\frac{E_{P}}{3}\left(\frac{1}{E_{g}}-\frac{1}{E_{g}+\Delta_{s o}}\right)+\frac{E_{P_{X}}}{3}\left(\frac{2}{E_{g}+E_{G C}+\Delta_{s o}+\Delta_{C}}-\frac{1}{E_{g}+E_{G C}}-\frac{1}{E_{g}+E_{G C}+\Delta_{C}}\right) \\
& \gamma_{\Delta 2}=\gamma_{2}-\frac{E_{P}}{12}\left(\frac{1}{E_{g}}-\frac{1}{E_{g}+\Delta_{s o}}\right)+\frac{E_{P_{X}}}{12}\left(\frac{2}{E_{g}+E_{G C}}-\frac{1}{E_{g}+E_{G C}+\Delta_{C}}-\frac{1}{E_{g}+E_{G C}+\Delta_{s o}+\Delta_{C}}\right) \\
& \gamma_{\Delta 3}=\gamma_{3}-\frac{E_{P}}{12}\left(\frac{1}{E_{g}}-\frac{1}{E_{g}+\Delta_{s o}}\right)-\frac{E_{P_{X}}}{12}\left(\frac{2}{E_{g}+E_{G C}}-\frac{1}{E_{g}+E_{G C}+\Delta_{C}}-\frac{1}{E_{G}+E_{G C}+\Delta_{s o}+\Delta_{C}}\right) .
\end{aligned}
$$

By adopting the numerical values of GaAs material parameters given in Ref. [68], more precisely $\left(\gamma_{1}, \gamma_{2}, \gamma_{3}\right)=$ $(7.03,2.33,3.03),\left(E_{g}, E_{G C}, \Delta_{s o}, \Delta_{C}\right)=(1.519 \mathrm{eV}, 3.0 \mathrm{eV}, 0.341 \mathrm{eV}, 0.18 \mathrm{eV}),\left(E_{P}, E_{P_{X}}\right)=(23.81 \mathrm{eV}, 15.79 \mathrm{eV})$, one obtains $\left(\gamma_{\Delta 1}, \gamma_{\Delta 2}, \gamma_{\Delta_{3}}\right)=(5.88,2.13,2.75)$. As a result, we get $m_{s o}=m_{0} / \gamma_{\Delta 1}=0.17 m_{0}$ which is very close to the recommended value of the split-off-hole effective mass given in Ref. [69]. Additionally, as $\left(\gamma_{\Delta 2}, \gamma_{\Delta_{3}}\right) \approx\left(\gamma_{2}, \gamma_{3}\right)$, we can assume $\mathfrak{C}_{\Delta} \approx \mathfrak{C}^{\mathfrak{i n t o}}$ the present analysis of the fine structure of bound excitons. 


\section{APPENDIX C: ELECTRON-HOLE EXCHANGE INTERACTION IN BULK}

Here, we present details of the calculations of various exchange energy terms of Sec. II D. For this purpose, we express the long-range part (also called nonanalytical part) and the short-range part (also known as the analytical part) of the exchange for one bulk semiconductor.

The band relevant part of the LR exchange Hamiltonian is defined as

$$
\underset{\substack{m n \\ m^{\prime} n^{\prime}}}{ }(\mathbf{q})=\sum_{i, j} \mathfrak{Q}_{\substack{m^{\prime} \mathcal{K} n \\ \mathcal{K} n^{\prime} m}}^{i j} q_{i} q_{j}
$$

in which

$$
\underset{\substack{\mathcal{K}_{n^{\prime} m} \\ \mathfrak{Q}^{\prime} \mathcal{K} n}}{i j}=\frac{\hbar^{2}}{m_{0}^{2}} \frac{\left\langle m^{\prime}\left|p_{i}\right| \mathcal{K} n^{\prime}\right\rangle\left\langle\mathcal{K} n\left|p_{j}\right| m\right\rangle}{\left(E_{m}^{0}-E_{n}^{0}\right)\left(E_{m^{\prime}}^{0}-E_{n^{\prime}}^{0}\right)},
$$

where $p_{i}\left(p_{j}\right)$ is the $i(j)$ component of the $\mathbf{p}$ momentum, $\mathcal{K}$ being the time-reversal operator, and $E_{\lambda}^{0}\left(\lambda=m, m^{\prime}, n, n^{\prime}\right)$ is the $\lambda$ th band energy.

We denote the conduction band Bloch wave function as $\left|C_{ \pm}\right\rangle=|1 / 2, \pm 1 / 2\rangle$, and the hole Bloch wave function as $\left|W_{1}\right\rangle=$ $|3 / 2,3 / 2\rangle,\left|W_{2}\right\rangle=|3 / 2,1 / 2\rangle,\left|W_{3}\right\rangle=|3 / 2,-1 / 2\rangle,\left|W_{4}\right\rangle=|3 / 2,-3 / 2\rangle,\left|W_{5}\right\rangle=|1 / 2,1 / 2\rangle,\left|W_{6}\right\rangle=|1 / 2,-1 / 2\rangle$.

For representing the $\mathfrak{Q}_{m^{\prime} n^{\prime}}^{i j}(\mathbf{q})$ matrix, we use the following electron-hole states $\left\{\begin{array}{c}\left|C_{+} W_{1}\right\rangle,\left|C_{-} W_{1}\right\rangle,\left|C_{+} W_{2}\right\rangle,\left|C_{-} W_{2}\right\rangle,\left|C_{+} W_{3}\right\rangle,\left|C_{-} W_{3}\right\rangle, \\ \left|C_{+} W_{4}\right\rangle,\left|C_{-} W_{4}\right\rangle,\left|C_{+} W_{5}\right\rangle,\left|C_{-} W_{5}\right\rangle,\left|C_{+} W_{6}\right\rangle,\left|C_{-} W_{6}\right\rangle\end{array}\right\}$ as basis. The matrix describing $\mathfrak{Q}_{m^{\prime} n^{\prime}}(\mathbf{q})$ is given by

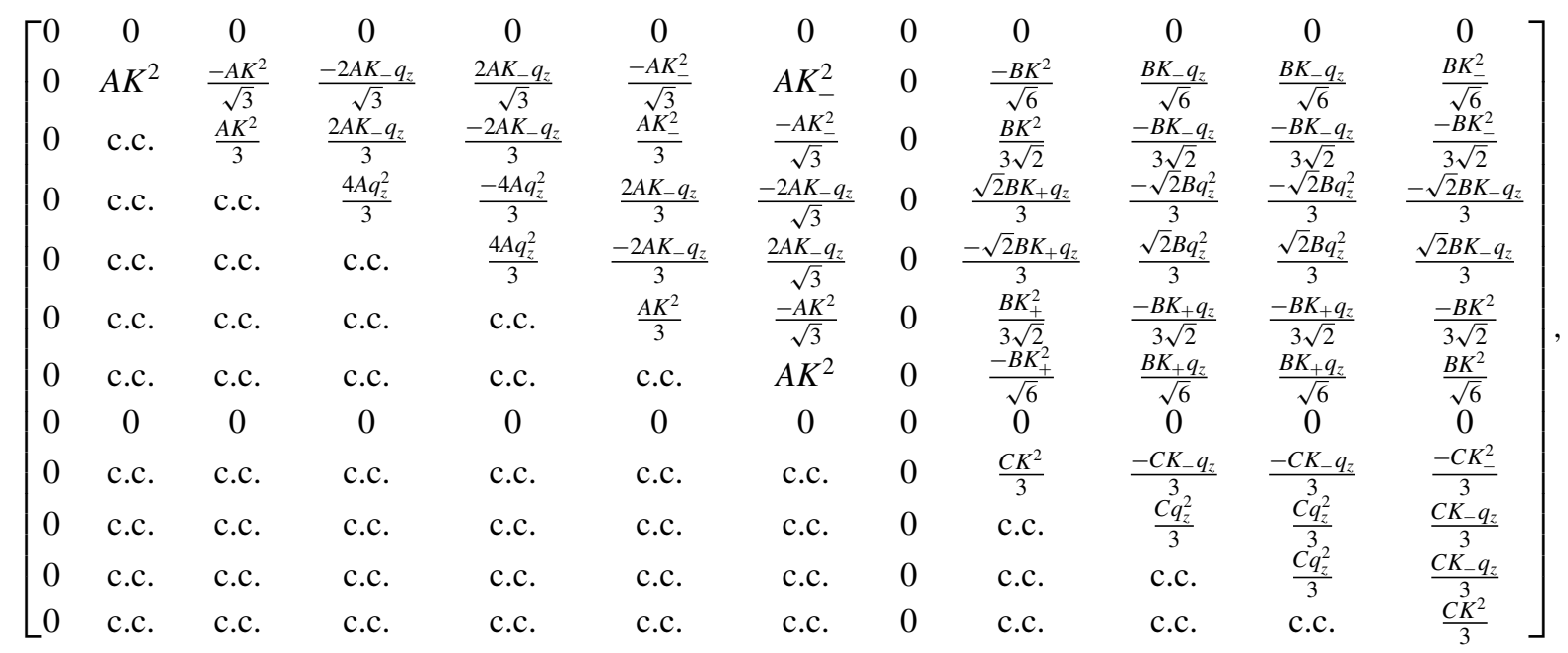

where c.c. denotes the complex conjugate, $K^{2}=\left(q_{x}^{2}+q_{y}^{2}\right), \quad K_{ \pm}=\left(q_{x} \pm i q_{y}\right), \quad A=\left(\hbar^{2} / 4 m_{0}\right)\left(E_{P} / E_{g}^{2}\right), \quad B=$ $\left(\hbar^{2} / 2 m_{0}\right)\left(E_{P} / E_{g}\left(E_{g}+\Delta_{s o}\right)\right)$, and $C=\left(\hbar^{2} / 2 m_{0}\right)\left(E_{P} /\left(E_{g}+\Delta_{s o}\right)^{2}\right)$. This matrix is identical to the one given by Eq. (8) of the Erratum [70] of Ref. [40].

Again using the basis of electron-hole states from the long-range part, the matrix describing the short-range exchange term in this $12 \times 12$ representation is given as

$$
\mathcal{H}_{m^{\prime} n^{\prime}}^{\mathrm{SR}}=K\left[\begin{array}{cccccccccccc}
0 & 0 & 0 & 0 & 0 & 0 & 0 & 0 & 0 & 0 & 0 & 0 \\
0 & 1 & \frac{-1}{\sqrt{3}} & 0 & 0 & 0 & 0 & 0 & -\sqrt{\frac{2}{3}} & 0 & 0 & 0 \\
0 & \text { c.c. } & \frac{1}{3} & 0 & 0 & 0 & 0 & 0 & \frac{\sqrt{2}}{3} & 0 & 0 & 0 \\
0 & 0 & 0 & \frac{2}{3} & \frac{-2}{3} & 0 & 0 & 0 & 0 & \frac{-\sqrt{2}}{3} & \frac{-\sqrt{2}}{3} & 0 \\
0 & 0 & 0 & c . c . & \frac{2}{3} & 0 & 0 & 0 & 0 & \frac{\sqrt{2}}{3} & \frac{\sqrt{2}}{3} & 0 \\
0 & 0 & 0 & 0 & 0 & \frac{1}{3} & \frac{-1}{\sqrt{3}} & 0 & 0 & 0 & 0 & \frac{-\sqrt{2}}{3} \\
0 & 0 & 0 & 0 & 0 & c . c . & 1 & 0 & 0 & 0 & 0 & \sqrt{\frac{2}{3}} \\
0 & 0 & 0 & 0 & 0 & 0 & 0 & 0 & 0 & 0 & 0 & 0 \\
0 & c . c . & c . c . & 0 & 0 & 0 & 0 & 0 & \frac{2}{3} & 0 & 0 & 0 \\
0 & 0 & 0 & c . c . & c . c . & 0 & 0 & 0 & 0 & \frac{1}{3} & \frac{1}{3} & 0 \\
0 & 0 & 0 & c . c . & c . c . & 0 & 0 & 0 & 0 & c . c . & \frac{1}{3} & 0 \\
0 & 0 & 0 & 0 & 0 & c . c . & c . c . & 0 & 0 & 0 & 0 & \frac{2}{3}
\end{array}\right]
$$


in which $K=D \delta\left(\mathbf{r}_{e}-\mathbf{r}_{h}\right) \delta\left(\mathbf{r}_{e}-\mathbf{r}_{e}^{\prime}\right) \delta\left(\mathbf{r}_{h}-\mathbf{r}_{h}^{\prime}\right), D$ being the short-range exchange constant. Similar matrix representation of the short-range part of exchange interaction is obtained by Tong and Wu (see Eq. (9) of Ref. [40]).

[1] P. Senellart, G. Solomon, and A. White, Nat. Nanotechnol. 12, 1026 (2017).

[2] D. Huber, M. Reindl, J. Aberl, A. Rastelli, and R. Trotta, J. Opt. 20, 073002 (2018).

[3] E. Biolatti, R. C. Iotti, P. Zanardi, and F. Rossi, Phys. Rev. Lett. 85, 5647 (2000).

[4] A. Imamoglu, D. D. Awschalom, G. Burkard, D. P. DiVincenzo, D. Loss, M. Sherwin, and A. Small, Phys. Rev. Lett. 83, 4204 (1999).

[5] S. Vichi, S. Bietti, A. Khalili, M. Costanzo, F. Cappelluti, L. Esposito, C. Somaschini, A. Fedorov, S. Tsukamoto, and P. Rauter, Nanotechnology 31, 245203 (2020).

[6] A. D. Yoffe, Adv. Phys. 50, 1 (2001).

[7] S. Fafard, K. Hinzer, S. Raymond, M. Dion, J. McCaffrey, Y. Feng, and S. Charbonneau, Science 274, 1350 (1996).

[8] N. Akopian, N. H. Lindner, E. Poem, Y. Berlatzky, J. Avron, D. Gershoni, B. D. Gerardot, and P. M. Petroff, Phys. Rev. Lett. 96, 130501 (2006).

[9] I. Schwartz, E. R. Schmidgall, L. Gantz, D. Cogan, E. Bordo, Y. Don, M. Zielinski, and D. Gershoni, Phys. Rev. X 5, 011009 (2015).

[10] I. Schwartz, D. Cogan, E. R. Schmidgall, L. Gantz, Y. Don, M. Zielinski, and D. Gershoni, Phys. Rev. B 92, 201201(R) (2015).

[11] E. Poem, Y. Kodriano, C. Tradonsky, N. H. Lindner, B. D. Gerardot, P. M. Petroff, and D. Gershoni, Nat. Phys. 6, 993 (2010).

[12] I. Kegel, T. H. Metzger, A. Lorke, and J. Peisl, J. Stangl, G. Bauer, J. M. Garcia, and P. M. Petroff, Phys. Rev. Lett. 85, 1694 (2000).

[13] A. Lemaître, G. Patriarche, and F. Glas, Appl. Phys. Lett. 85, 3717 (2004).

[14] N. Koguchi, S. Takahashi, and T. Chikyow, J. Crystal. Growth 111, 688 (1991).

[15] Zh. M. Wang, B. L. Liang, K. A. Sablon, and G. J. Salamo, Appl. Phys. Lett. 90, 113120 (2007).

[16] Ch. Heyn, A. Stemmann, T. Köppen, Ch. Strelow, T. Kipp, M. Grave, S. Mendach, and W. Hansen, Appl. Phys. Lett. 94, 183113 (2009).

[17] Y. H. Huo, A. Rastelli, and O. G. Schmidt, Appl. Phys. Lett. 102, 152105 (2013).

[18] Y. H. Huo, V. Krapek, A. Rastelli, and O. G. Schmidt, Phys. Rev. B 90, 041304(R) (2014).

[19] S. Kiravittaya, M. Benyoucef, R. Zapf-Gottwick, A. Rastelli, and O. G. Schmidt, Appl. Phys. Lett. 89, 233102 (2006).

[20] E. Zallo, P. Atkinson, L. Wang, A. Rastelli, and O. G. Schmidt, Phys. Status Solidi B 249, 702 (2012).

[21] Y. H. Huo, V. Krapek, O. G. Schmidt, and A. Rastelli, Phys. Rev. B 95, 165304 (2017).

[22] A. Pankratov, Ph.D. thesis, Sorbonne University, 2017, https: //tel.archives-ouvertes.fr/tel-01589000/document.

[23] J. D. Plumhof, R. Trotta, V. Křápek, E. Zallo, P. Atkinson, S. Kumar, A. Rastelli, and O. G. Schmidt, Phys. Rev. B 87, 075311 (2013).
[24] L. C. Lew, Yan Voon and M. Willatzen, The k.p Method : Electronic Properties of Semiconductors (Springer-Verlag, Berlin, Heidelberg, 2009).

[25] H. Fu, L-W. Wang, and A. Zunger, Phys. Rev. B 59, 5568 (1999).

[26] J-W Luo, G. Bester, and A. Zunger, Phys. Rev. B 79, 125329 (2009).

[27] P. A. Labud, A. Ludwig, A. D. Wieck, G. Bester, and D. Reuter, Phys. Rev. Lett. 112, 046803 (2014).

[28] H. Bui, A. Karpulevich, and G. Bester, Phys. Rev. B 101, 115414 (2020).

[29] J-M. Jancu, R. Scholz, F. Beltram, and F. Bassani, Phys. Rev. B 57, 6493 (1998).

[30] M. Zieliński, M. Korkusiński, and P. Hawrylak, Phys. Rev. B 81, 085301 (2010).

[31] M. Zieliński, Phys. Rev. B 88, 115424 (2013).

[32] M. Zielinski, Phys. Rev. B 99, 205402 (2019).

[33] E. Kadantsev and P. Hawrylak, Phys. Rev. B 81, 045311 (2010).

[34] D. Huber, B. U. Lehner, D. Csontosová, M. Reindl, S. Schuler, S. F. Covre da Silva, P. Klenovský, and A. Rastelli, Phys. Rev. B 100, 235425 (2019).

[35] D. Csontosová and P. Klenovský, Phys. Rev. B 102, 125412 (2020).

[36] Z. Trabelsi, M. Yahyaoui, K. Boujdaria, M. Chamarro, and C. Testelin, J. Appl. Phys. 121, 245702 (2017).

[37] Z. Trabelsi, M. Yahyaoui, S. Ben Radhia, K. Boujdaria, E. Zallo, O. G. Schmidt, P. Atkinson, M. Chamarro, and C. Testelin, J. Lum. 197, 47 (2018).

[38] G. E. Pikus and G. L. Bir, Zh. Eksp. Teor. Fiz. 60, 195 (1971) [Sov. Phys. JETP 33, 108 (1973)].

[39] M. M. Denisov and V. P. Makarov, Phys. Status Solidi B 56, 9 (1973).

[40] H. Tong and M. W. Wu, Phys. Rev. B 83, 235323 (2011).

[41] A. I. Tartakovskii, M. N. Makhonin, I. R. Sellers, J. Cahill, A. D. Andreev, D. M. Whittaker, J-P. R. Wells, A. M. Fox, D. J. Mowbray, M. S. Skolnick, K. M. Groom, M. J. Steer, H. Y. Liu, and M. Hopkinson, Phys. Rev. B 70, 193303 (2004).

[42] J. W. Luo and A. Zunger, Phys. Rev. B 84, 235317 (2011).

[43] R. Singh and G. Bester, Phys. Rev. B 84, 241402(R) (2011).

[44] M. Zielinski, Y. Don, and D. Gershoni, Phys. Rev. B 91, 085403 (2015).

[45] T. Takagahara, Phys. Rev. B 62, 16840 (2000).

[46] M. Korkusinski and P. Hawrylak, Phys. Rev. B 87, 115310 (2013).

[47] M. Gawełczyk, M. Syperek, A. Maryński, P. Mrowiński, L. Dusanowski, K. Gawarecki, J. Misiewicz, A. Somers, J. P. Reithmaier, S. Höfling, and G. Sęk, Phys. Rev. B 96, 245425 (2017).

[48] M. Gawełczyk, P. Wyborski, P. Podemski, J. P. Reithmaier, S. Höfling, and G. Sęk, Phys. Rev. B 100, 241304(R) (2019).

[49] M. Zieliński, Sci. Rep. 10, 13542 (2020). 
[50] Y. Shinozuka and M. Matsuura, Phys. Rev. B 28, 4878 (1983).

[51] M. Bayer, S. N. Walck, T. L. Reinecke, and A. Forchel, Phys. Rev. B 57, 6584 (1998).

[52] R. L. Greene, K. K. Bajaj, and D. E. Phelps, Phys. Rev. B 29, 1807 (1984).

[53] P. O. Löwdin, J. Chem. Phys. 19, 1396 (1951).

[54] J. M. Luttinger and W. Kohn, Phys. Rev. 97, 869 (1955).

[55] B. J. BenDaniel and C. B. Duke, Phys. Rev. 152, 683 (1966).

[56] O. Zitouni, K. Boujdaria, and H. Bouchriha, Semicond. Sci. Technol. 20, 908 (2005).

[57] W. Ekardt, K. Lösch, and D. Bimberg, Phys. Rev. B 20, 3303 (1979).

[58] E. Blackwood, M. J. Snelling, R. T. Harley, S. R. Andrews, and C. T. B. Foxon, Phys. Rev. B 50, 14246 (1994).

[59] M. Bayer, G. Ortner, O. Stern, A. Kuther, A. A. Gorbunov, A. Forchel, P. Hawrylak, S. Fafard, K. Hinzer, T. L. Reinecke, S. N. Walck, J. P. Reithmaier, F. Klopf, and F. Schäfer, Phys. Rev. B 65, 195315 (2002).
[60] Following the convention of Ref. [21], one has the following relationships with the FSS parameters $\left(\delta_{0}, \delta_{1}, \delta_{2}\right)$ of Ref. [22]: $\delta_{\mathrm{BB}}=\delta_{1}, \delta_{\mathrm{DD}}=\delta_{2}, \delta_{\mathrm{BD}}=\delta_{0}-\left(\delta_{1}+\delta_{2}\right) / 2$.

[61] J-W. Luo, R. Singh, A. Zunger, and G. Bester, Phys. Rev. B 86, 161302(R) (2012).

[62] A. Schliwa, M. Winkelnkemper, and D. Bimberg, Phys. Rev. B 79, 075443 (2009).

[63] D. Fuster, Y. González, and L. González, Nanoscale Res. Lett. 9, 309 (2014).

[64] J. W. Luo, A. Franceschetti, and A. Zunger, Phys. Rev. B 79, 201301(R) (2009).

[65] J. W. Luo, G. Bester, and A. Zunger, New J. Phys. 11, 123024 (2009).

[66] K. Boujdaria, S. Ridene, and G. Fishman, Phys. Rev. B 63, 235302 (2001).

[67] H. Meyer and U. Rössler, Phys. Rev. B 44, 9048 (1991).

[68] I. Saïdi, S. Ben Radhia, and K. Boujdaria, J. Appl. Phys. 107, 043701 (2010).

[69] I. Vurgaftman and J. R. Meyer, J. Appl. Phys. 89, 5815 (2001). [70] H. Tong and M. W. Wu, Phys. Rev. B 84, 039903(E) (2011). 